\title{
Functional neuroimaging of sensorimotor cortices in postmenopausal women with type II diabetes
}

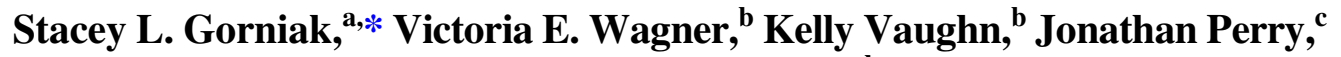 \\ Lauren Gulley Cox, ${ }^{\text {a }}$ Arturo E. Hernandez, ${ }^{\text {b }}$ and Luca Polloninic \\ ${ }^{a}$ University of Houston, Department of Health and Human Performance, Houston, Texas, \\ United States \\ ${ }^{b}$ University of Houston, Department of Psychology, Houston, Texas, United States \\ ${ }^{\mathrm{c}}$ University of Houston, Department of Engineering Technology, Houston, Texas, United States
}

\begin{abstract}
Significance: Deficits in sensorimotor function in persons with type II diabetes mellitus (PwDM) have traditionally been considered a result of peripheral nerve damage. Emerging evidence has suggested that factors outside of nerve damage due to type II diabetes mellitus, such as impaired hemodynamic function, contribute significantly to both sensory and motor deficits in PwDM.
\end{abstract}

Aim: The focus of the current study was to evaluate functional cortical hemodynamic activity during sensory and motor tasks in PwDM.

Approach: Functional near-infrared spectroscopy was used to monitor oxyhemoglobin ( $\mathrm{HbO})$ and deoxyhemoglobin ( $\mathrm{HbR}$ ) across the cortex during sensory and motor tasks involving the hands.

Results: Decline in $\mathrm{HbO}$ across sensory and motor regions of interest was found in PwDM with simultaneous deficits in manual motor tasks, providing the first evidence of functional cortical hemodynamic activity deficits relating to motor dysfunction in PwDM. Similar deficits were neither specifically noted in HbR nor during evaluation of sensory function. Health state indices, such as $\mathrm{A}_{1 \mathrm{c}}$, blood pressure, body mass index, and cholesterol, were found to clarify group effects.

Conclusions: Further work is needed to clarify potential sex-based differences in PwDM during motor tasks as well as the root of reduced cortical $\mathrm{HbO}$ indices but unchanged $\mathrm{HbR}$ indices in PwDM.

(C) The Authors. Published by SPIE under a Creative Commons Attribution 4.0 Unported License. Distribution or reproduction of this work in whole or in part requires full attribution of the original publication, including its DOI. [DOI: 10.1117/1.NPh.7.3.035007]

Keywords: functional near-infrared spectroscopy; aging; cortical oxygenation; motor dysfunction; tactile sensation.

Paper 20037R received Apr. 20, 2020; accepted for publication Aug. 14, 2020; published online Sep. 2, 2020.

\section{Introduction}

Nearly $24 \%$ of the 40 million individuals in the United States over the age of 60 are currently living with type II diabetes mellitus (DM). ${ }^{1}$ Persons with DM (PwDM) experience decline in hand/finger sensorimotor function as compared to healthy individuals; ${ }^{2-4}$ however, selfawareness of these changes is low. ${ }^{5}$ Reduced functional hand use has been associated with a loss of independent living and reduced quality of life in PwDM. ${ }^{6,7}$ Tactile dysfunction due to peripheral neuropathy (PN) has been implicated as the primary cause of motor deficits in $\mathrm{PwDM} ;{ }^{8-11}$ however, our recent work has demonstrated that motor changes in PwDM occur independent of tactile impairment, unrelated to disease duration and severity. ${ }^{12}$ Our data point

*Address all correspondence to Stacey L. Gorniak, E-mail: sgorniak@uh.edu 
toward other factors, such as alterations in motor unit structure-function and reduced hemodynamic function of muscle and the cortex as underlying mechanisms for these sensorimotor changes. ${ }^{13}$ Due to the confluence of multiple systemic changes in the body in PwDM, the contribution of impaired or altered cortical function to sensorimotor dysfunction prior to PN diagnosis in PwDM is fully plausible.

Altered hemodynamic responses due to both micro- and macrovascular changes have been implicated as a potential source of global motor changes in PwDM. ${ }^{14}$ Specifically, endothelial dysfunction within dermal and muscle tissues in adult PwDM has been related to altered motor behaviors, including abnormal force production. ${ }^{13,15,16}$ Currently, it is unclear whether hemodynamic function of cortical tissues, as evidenced by abnormal cortical activation patterns, also occurs in PwDM. Correspondingly, our hypothesis is that altered hemodynamic function of the cortex occurs during tactile sensory and manual motor tasks. Further, these altered hemodynamic patterns may directly relate to poor tactile detection thresholds and motor performance in PwDM. In particular, postmenopausal women may experience significant deterioration of both hemodynamic function and resultant sensorimotor behaviors given the disproportionate risk of cardiovascular complications in women with DM as compared to men with DM and persons without DM. ${ }^{17,18}$ Accordingly, the focus of this study was to evaluate changes in cortical oxygenation indices of postmenopausal women both with and without DM during (1) tactile stimulation of the hands and (2) during manual isometric submaximal force production tasks.

We expected to see between-group changes in cortical oxygenation indices, such as oxygenated hemoglobin $(\mathrm{HbO})$ and deoxygenated or reduced hemoglobin $(\mathrm{HbR})$, during tactile stimulation of the hands and during isometric submaximal force production tasks (Hypothesis \#1). Additionally, based on our previous work, we expected to see between-group differences in tactile sensitivity and in the temporal structure of force variability (Hypothesis \#2). No hypotheses regarding changes in cortical hemodynamic function with disease state were developed $a$ priori, as investigation of cortical hemodynamic function with disease state was an exploratory aim of this study. To examine these hypotheses, we measured cortical hemodynamic activity with functional near-infrared spectroscopy (fNIRS) alongside individual task performance with the goals of (1) detecting differences between PwDM and controls and (2) evaluating the relationship between cortical hemodynamic activity and task performance.

\section{Materials and Methods}

\subsection{Participants}

Twenty-one self-declared right-handed postmenopausal women with DM and 21 self-declared right-handed age- and sex-matched healthy controls volunteered to participate in the study, see Table 1 for demographics. Handedness was confirmed by the Edinburgh Inventory, ${ }^{19}$ ranging from a laterality quotient (LQ) of -100 (strong left-handedness) to +100 (strong right-handedness). Participants had an LQ average of +88 and had no previous history of trauma to the upper limbs. Study participants were excluded if they reported a history of neurological and/or musculoskeletal disorders (Parkinson disease, Huntington's disease, polio, multiple sclerosis, stroke, traumatic brain injury, carpal tunnel syndrome, rheumatoid arthritis, monoclonal gammopathy of undetermined significance, paraproteinemic demyelinating neuropathy, myasthenia gravis), a history of amputation, a history of major surgical intervention to the upper extremity, or hereditary or compression neuropathies. In accordance with the Declaration of Helsinki, participants provided informed consent according to the regulations established by the Institutional Review Board at the University of Houston (protocol \#15615-01). Data collection processes failed on five participants (e.g., a reliable fNIRS signal was not detected). These were the following participants: controls 2, 7, 10, and 21 as well as PwDM 19. This resulted in a sample size of 17 controls and 20 PwDM. Data from those participants have been excluded from these analyses. Post-hoc power analyses using G*Power 3.1.94 indicated that the primary effect size found $(d=0.951)$ in $\mathrm{HbO}$ data with the sample sizes of 17 controls and $20 \mathrm{PwDM}$ provided for a post-hoc power of 0.88 , well above the 0.8 power threshold for traditional power analyses. 
Gorniak et al.: Functional neuroimaging of sensorimotor cortices in postmenopausal women...

Table 1 Demographic and clinical characteristics of DM participants.

\begin{tabular}{|c|c|c|c|c|c|c|c|c|}
\hline $\begin{array}{l}\text { Participant } \\
\#\end{array}$ & $\begin{array}{c}\text { Age } \\
\text { (years) }\end{array}$ & $\begin{array}{l}\text { Menopausal } \\
\text { age (years) }\end{array}$ & $\begin{array}{c}\text { BMI } \\
\left(\mathrm{kg} / \mathrm{m}^{2}\right)\end{array}$ & $\begin{array}{c}\text { DM } \\
\text { duration } \\
\text { (months) }\end{array}$ & $\begin{array}{l}A_{1 c} \\
(\%)\end{array}$ & $\begin{array}{c}\text { Total } \\
\text { cholesterol } \\
(\mathrm{mg} / \mathrm{dL})\end{array}$ & $\begin{array}{l}\text { Systole } \\
(\mathrm{mmHg})\end{array}$ & $\begin{array}{l}\text { Diastole } \\
(\mathrm{mmHg})\end{array}$ \\
\hline 1 & 63 & 50 & 27.4 & 60 & 6.7 & - & 151 & 81 \\
\hline 2 & 79 & 45 & 28.3 & 144 & 7.9 & - & 155 & 75 \\
\hline $3^{a}$ & 65 & 50 & 40.7 & 120 & 7.1 & - & 145 & 97 \\
\hline $4^{b}$ & 66 & 50 & 29.3 & 186 & 8.7 & 199 & 111 & 62 \\
\hline $5^{a}$ & 64 & 40 & 44.1 & 60 & 6.2 & 109 & 180 & 91 \\
\hline 6 & 60 & 50 & 37.5 & 387 & 10.4 & 224 & 161 & 78 \\
\hline $7^{\mathrm{a}}$ & 60 & 55 & 33.7 & 245 & 8 & 143 & 130 & 70 \\
\hline 8 & 57 & 49 & 36.9 & 41 & 8.6 & 176 & 130 & 88 \\
\hline 9 & 73 & 60 & 25.3 & 201 & 6.8 & 125 & 167 & 78 \\
\hline 10 & 68 & 23 & 31.8 & 168 & 5.7 & 219 & 164 & 89 \\
\hline 11 & 70 & 45 & 26.9 & 200 & 6.1 & 266 & 130 & 71 \\
\hline 12 & 62 & 38 & 32.4 & 36 & 6.2 & 189 & 124 & 70 \\
\hline 13 & 67 & 45 & 30.2 & 1 & 8 & 144 & 158 & 97 \\
\hline $14^{\mathrm{a}, \mathrm{b}}$ & 66 & 45 & 31.4 & 262 & 6.3 & 175 & 142 & 75 \\
\hline $15^{\mathrm{a}}$ & 69 & 55 & 42.3 & 298 & 8.4 & 185 & 139 & 63 \\
\hline 16 & 58 & 51 & 32.8 & 95 & 7.4 & 143 & 153 & 89 \\
\hline $17^{a}$ & 55 & 27 & 38.6 & 385 & 7.4 & 126 & 133 & 68 \\
\hline 18 & 67 & 25 & 30.5 & 1 & 7.7 & 183 & 148 & 73 \\
\hline $19^{a}$ & 71 & 52 & 42.9 & 196 & 8.5 & 173 & 105 & 60 \\
\hline 20 & 69 & 27 & 36.3 & 149 & 8.7 & 187 & 202 & 100 \\
\hline 21 & 60 & 37 & 30.1 & 1 & 6.7 & 183 & 179 & 111 \\
\hline Mean & 65 & 43 & 33.8 & 154 & 7.5 & 175 & 148 & 80 \\
\hline SD & 6 & 11 & 5.6 & 117 & 1.2 & 39 & 23 & 14 \\
\hline Controls & $67 \pm 6$ & $50 \pm 7$ & $24.1 \pm 4.5$ & $\mathrm{~N} / \mathrm{A}$ & $5.3 \pm 0.3$ & $200 \pm 43$ & $147 \pm 21$ & $86 \pm 14$ \\
\hline
\end{tabular}

Note: SD, standard deviation; - , data collection failure.

andicates a clinical diagnosis of diabetic peripheral neuropathy.

${ }^{\mathrm{b}}$ Indicates a history of Prempro Rx (in addition to three control participants).

\subsection{Health Status Data}

Blood pressure, cholesterol, and glycated hemoglobin $\left(\mathrm{A}_{1 \mathrm{c}}\right)$ values were assessed for all study participants onsite. Cholesterol and $\mathrm{A}_{1 \mathrm{c}}$ values were assessed using a commercially available point of care evaluation kit (Cardiocheck+ and $\mathrm{A}_{1 \mathrm{c}}$ Now+ kits, PTS Diagnostics, Indianapolis, Indiana). Blood pressure was measured using a commercially available device (Omron Intellisense 10 series Blood Pressure Monitor, Model BP785, Bannockburn, Illinois). The presence of PN (PN status) was determined by abnormalities on either clinical examination or electromyography/nerve conduction velocity (EMG/NCV) testing (per physician). A brief menopause questionnaire was also administered regarding several aspects of menopausal 
characteristics (e.g., age at onset of menopause, hormone replacement therapy history, etc.). All study participants declared themselves to be postmenopausal with 11 participants claiming a history of hormone replacement therapy (five with a history of Prempro use).

\subsection{Tactile Sensory Evaluation}

The Semmes-Weinstein monofilament test was used to evaluate tactile sensation of the dominant hand. ${ }^{20}$ Monofilament testing sites included the tip of the thumb/digit 1 (median nerve), the tip of digit $5 /$ hypothenar eminence (ulnar nerve), and dorsal aspect of the thumb (radial nerve). During the test, participants kept their eyes closed and verbally indicated if and where they perceived monofilament touch. The monofilament size was increased until the subject was able to detect its touch a minimum of two times at the same location.

\subsection{Maximal Force Production}

Participants were asked to perform a series of force production tasks consisting of precision pinching (use of digits 1 and 2) at maximal strength using the dominant (right) hand. Maximal pinch evaluation was conducted using a Biometrics Pinch Dynamometer and wireless DataLOG system (Precision Pinchmeter model P200, DataLOG model MWX8, Biometrics Ltd, Newport, UK). Three trials were collected with the dominant hand, and average maximal force production values (MVC) were calculated. During testing, the P200 device was placed 16 in. anterior to the patient torso, 6 in. away from the midline of the body toward the dominant hand. The wrist orientation was such that the hand was in a neutral position during testing. ${ }^{4,5,21}$ During testing, the P200 device was held vertically with the nondominant hand.

\subsection{Experimental Tasks}

Each subject was asked to perform a series of sensory simulation and manual motor tasks executed in a specific order (all sensory tasks prior to all motor tasks) and interleaved by 30-s periods of rest, see Fig. 1 for details. Presentation of visual stimuli, timing, and task-specific synchronization TTL signals was controlled via E-prime 2.0 (Psychology Software Tools, Inc., Sharpsburg, Pennsylvania). During sensory stimulation tasks, digits 1 and 2 of the dominant (right) hand were stimulated with a tendon vibrator device (VB115 Techno Concept Tendon Vibrator Kits, Techno Concept, France) at an amplitude of $1 \mathrm{~mm}$ at two frequencies: 25 and $115 \mathrm{~Hz}$. Stimulation frequency presentation was block randomized. Three stimulation trials (lasting $30 \mathrm{~s}$ each with $30 \mathrm{~s}$ washout periods in between) were presented per stimulation frequency.

During the manual motor task, participants used a precision pinch grip to exert an isometric force against a set of force transducers, see below for details. Participants were instructed to match their pinch force to the target force line as accurately as possible. Two different force

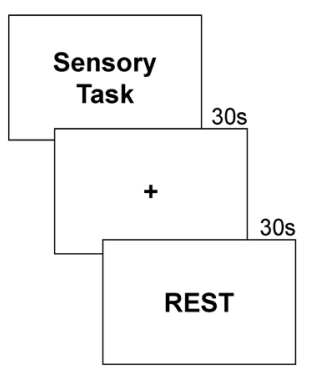

$25 \mathrm{~Hz}$ and $115 \mathrm{~Hz}$ stimulus presentation block randomized

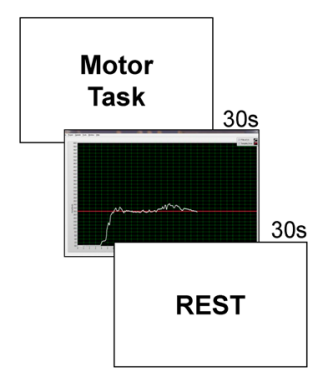

$15 \%$ and $40 \%$ MVC force production block randomized

Fig. 1 Illustration of experimental stimuli during the vibrotactile stimulation and motor performance tasks during fNIRS testing. Subjects viewed a fixation cross during vibrotactile stimulation blocks; they viewed real-time feedback on their force production during motor blocks. The order of stimulus presentation was block randomized within stimulation type. Sensory stimulation tasks always occurred prior to motor tasks. 
levels were tested for the dominant (right) hand (15\% MVC and 40\% MVC), see Sec. 2.5.1 for details. Three trials of $30 \mathrm{~s}$ each were performed with $30 \mathrm{~s}$ of rest/washout periods between each block. Force level order (15\% or $40 \%$ MVC) was block randomized.

\subsubsection{Submaximal force production task}

Briefly, the task involved using digits 1 and 2 in a precision pinch grip to produce a constant level of pinch force with feedback from a computer screen. All forces and moments of force produced were recorded simultaneously using two identical six-component force-moment transducers (Nano-25 transducers; ATI Industrial Automation, Garner, North Carolina). Instrument details have been published. ${ }^{4,5,21}$ No contact of either transducer was permitted prior to trial onset. Two different force production levels were tested: $15 \%$ and 40\% MVC. MVC values for pinch forces were determined from maximal dynamometry testing (see above). Each participant performed three trials for each condition, lasting $30 \mathrm{~s}$ each. Force production order was block randomized. Subjects were not asked to bear the weight of the object, instead all kinetic testing devices were suspended so that participants could easily touch and exert force on the devices without movement of the hand/fingers/forearm (SnakeClamp, Riner, Virginia).

Transducer signals were amplified and multiplexed using ATI hardware prior to being routed to an analog-to-digital converter (via cDAQ-9174 chassis and NI-9205 input modules, National Instruments, Austin, Texas). A customized Labview program (National Instruments) was used for data acquisition and customized MATLAB ${ }^{\circledR}$ (Mathworks Inc., Natick, Massachusetts) programs were written for data processing. Signals were sampled at $100 \mathrm{~Hz}$ and low-pass filtered at $10 \mathrm{~Hz}$ using a second-order, zero-lag Butterworth filter. ${ }^{4,5,21}$ The force data of interest consisted of the final $25 \mathrm{~s}$ of each trial. ${ }^{4,5,21}$ Participants were required to reach and maintain the indicated force production level within the first $5 \mathrm{~s}$ of each trial. Force data of the three trials per condition were averaged for kinetic analyses.

\subsubsection{Kinetic analyses}

Submaximal data were analyzed with respect to both linear and nonlinear measures. Linear measures of performance included root mean square error (RMSE) of the force output relative to the target and the coefficient of variation $(\mathrm{CV})$. The structure of force output variability was quantified via approximate entropy (ApEn) and detrended fluctuation analysis (DFA). Center of pressure (COP) data was determined via analysis of the measured force and torque data, as described by Zatsiorsky. ${ }^{22}$ The mean area of the COP was calculated by using an $85 \%$ best fit ellipse to the COP data using principal component analysis. ${ }^{23}$ The mean area of this ellipse was used as a measure of consistent digit placement and fingertip roll throughout the duration of skin to object contact in manual tasks. ${ }^{24}$

\subsection{Cortical Hemodynamics Measurements}

Cortical hemodynamics were measured with a continuous-wave fNIRS instrument (NIRScout, NIRx Technologies, Glen Head, New York) via 16 optical emitters and 16 optical detectors. Each emitter consisted of a dual-wavelength LED (central wavelengths: 760 and $850 \mathrm{~nm}$ ) directly coupled to the scalp. Each detector was a silicon photodiode collecting backscattered light from the scalp via an optical fiber. The geometrical layout of optical emitters and detectors (collectively referred to as optodes) is shown in Fig. 2(a), alongside the corresponding sensitivity map of the optical probing on the cerebral cortex [Fig. 2(b)] estimated with Monte Carlo-based simulation of photon migration in AtlasViewer. ${ }^{26}$ This configuration resulted in 28 optical channels (i.e., emitter-detector pairings) that interrogated the prefrontal, motor, and somatosensory cortices bilaterally. The geometrical distance between optode pairings ranged from 26 to $37 \mathrm{~mm}$, ensuring the interrogation of the cerebral cortex in all optical channels. ${ }^{27}$

Raw optical signals were collected continuously throughout the experiment at a frequency of $3.91 \mathrm{~Hz}$ from all channels at both wavelengths and were subsequently converted to optical density (i.e., logarithm of the raw intensity) and then to concentration changes of $\mathrm{HbO}$ and $\mathrm{HbR}$ compared to a zeroed baseline according to the modified Beer-Lambert law. ${ }^{28,29}$ For each 
(a)

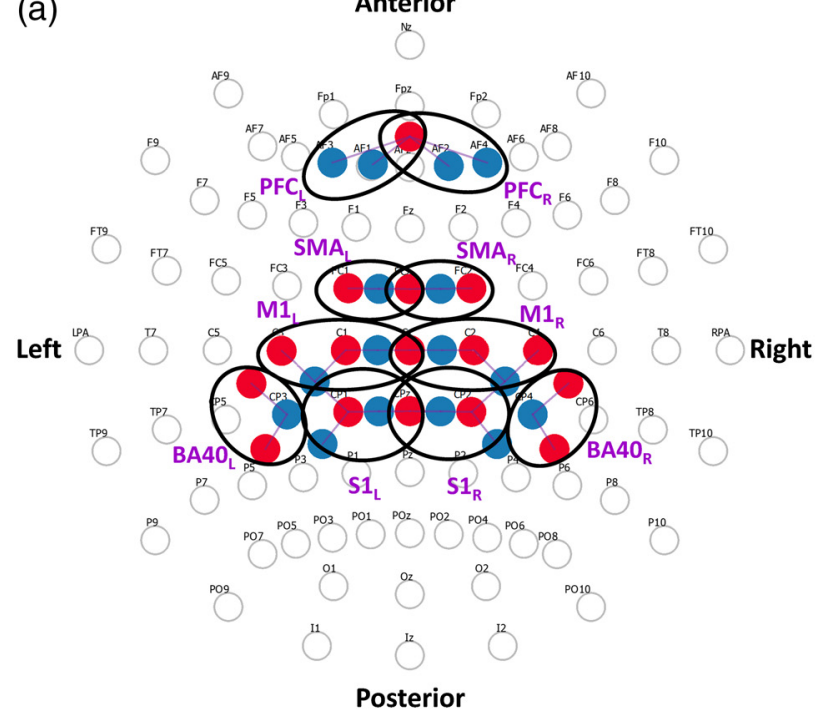

(b)

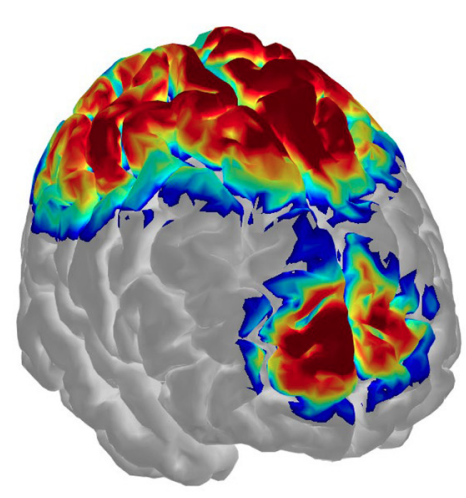

Fig. 2 Cortical fNIRS layout and sensitivity map. (a) Geometrical layout of sources (red) and detectors (blue) with respect to the international 10-10 electroencephalogram (EEG) system. ${ }^{25}$ Bold black ovals denote the ROls, which are subsequently labeled nearby in purple boldface. ROI hemisphere is denoted in subscript. ROls included PFC, SMA, M1, S1, and BA40. Hemisphere side as well as anterior and posterior of the cranium are noted. (b) Correspondent sensitivity map overlaid onto the Colin27 brain model. Sensitivity computed and displayed with AtlasViewer. ${ }^{26}$

channel, $\mathrm{HbO}$ and $\mathrm{HbR}$ measurements were analyzed separately with a general linear model approach that estimated the scalar weight coefficient (a.k.a., beta weight ${ }^{30}$ ) of the canonical hemodynamic response that best least-squared fitted the measured hemodynamic response. The general linear model, based on an autoregressive iteratively reweighted least-squares approach, is described in detail in Ref. 31. Of relevance, this method permits correction of motion artifacts and serial correlations within the algorithm [i.e., does not require one to explicitly bandpass filter or apply corrective actions to detect and discard extracerebral, unwanted components, such as systemic oscillations (i.e., cardiac and respiration) and motion artifacts]. ${ }^{30,32}$ For each subject, we considered channels as hemodynamically active if their weight coefficient was statistically different from zero at the significance level of $5 \%$.

At the group level, we used a mixed linear model to estimate the weighting coefficient of all channels in order to determine which of them were hemodynamically active at a statistically significant level. We considered the interaction between the experimental condition (sensory stimulation at high, sensory stimulation at low, motor performance at high force level, and motor performance at low force level) and the group (DM versus control) as the fixed effect contributing to the weight coefficient, while the magnitude of the coefficient of individual subjects was considered as a random effect. Both $\mathrm{HbO}$ and $\mathrm{HbR}$ were fitted with a positive double-gamma hemodynamic response function (HRF) (canonical HRF), hence, a negative $t$-value for either $\mathrm{HbO}$ or $\mathrm{HbR}$ signifies a decrease in respective concentration.

We grouped optical channels into 10 bilateral (right and left) regions of interest (ROIs): prefrontal cortex (PFC), supplementary motor area (SMA), primary motor cortex (M1), primary sensory cortex (S1), and Broadmann Area 40 (BA40), as depicted in Fig. 2(a). We computed individual-level ROI level statistics (weight coefficient, $t$-value, and $p$-value).

\subsection{Statistical Analysis}

The data are presented as means \pm standard errors (SE). For $\mathrm{HbO}$ and HbR, statistically significant individual-level ROI $t$-scores were compared between "groups" using mixed model analyses of covariance (ANCOVAs) via SPSS 25 (IBM Corporation, Armonk, New York). Between-subject primary factors were group (two levels: DM versus controls). Within-subject factors included "hemisphere" (two levels for the cortex: left and right) and "ROI" (five levels: 
$1=$ PFC, 2 = SMA, 3 = M1, $4=\mathrm{S} 1$, and 5 = BA40). For monofilament data, main factors included group and nerve (three levels: one level each for the median, radial, and ulnar nerves). For submaximal force production data, main factors included group and level (two levels: $15 \%$ and $40 \% \mathrm{MVC}$ ). Evaluation of health state covariates was done to control for health state variability both within and across the two sample groups. ANCOVAs included health state covariates of $\mathrm{A}_{1 \mathrm{c}}$, systolic and diastolic blood pressures, low-density lipoprotein cholesterol, high-density lipoprotein cholesterol, disease duration, menopausal age, body mass index (BMI), PN status (via indicator variable), history of hormone replacement therapy (via indicator variable), and history of treatment with Prempro (via indicator variable). Covariates were selected via automatic linear modeling (ALM) using forward stepwise selection functions in SPSS. Standard SPSS ALM parameters were set at outliers automatically trimmed, single standard model creation, 95\% confidence intervals set, use of the AICc information criterion function, and the replication feature enabled. ALM models were run for each measure of interest in order to investigate the influence of health state measures on all behavioral data. In the event of significant covariates determined via ALM and ANCOVA, follow-up correlation analyses were performed between the health state covariate and the measured behavior. Monofilament data were $\log$ transformed due to nonlinearity. ${ }^{33}$ Nontransformed values are shown in figures to avoid reader confusion.

\section{Results}

\subsection{Tactile Evaluation}

A significant difference in tactile detection thresholds was not specifically confirmed between groups $(p=0.159)$; however, the addition of covariates with the statistical model led to a between group difference that approached significance $(p=0.098)$. Tactile detection threshold values did differ among all tested sites [nerve: $F_{2,195}=4.52, p<0.05$, Fig. 3(a)] with a significant difference in the median and radial nerve tactile detection thresholds. Tactile function was generally worse in persons with higher BMI, higher $\mathrm{A}_{1 \mathrm{c}}$, and in those diagnosed with PN, see Table 2 for covariate results.

(a)
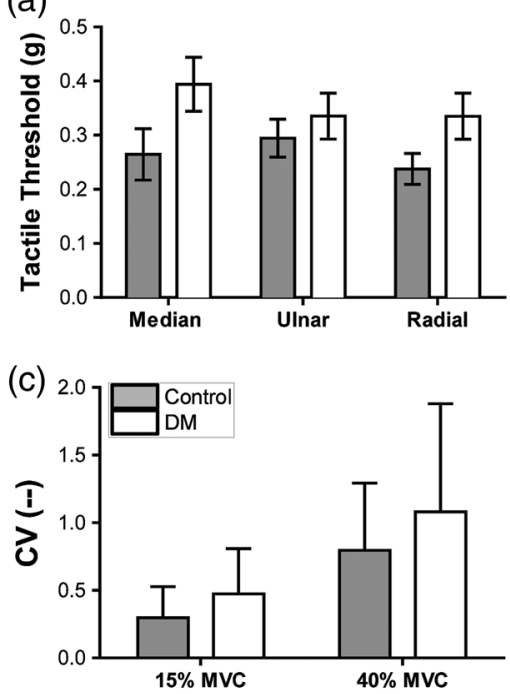

(b)
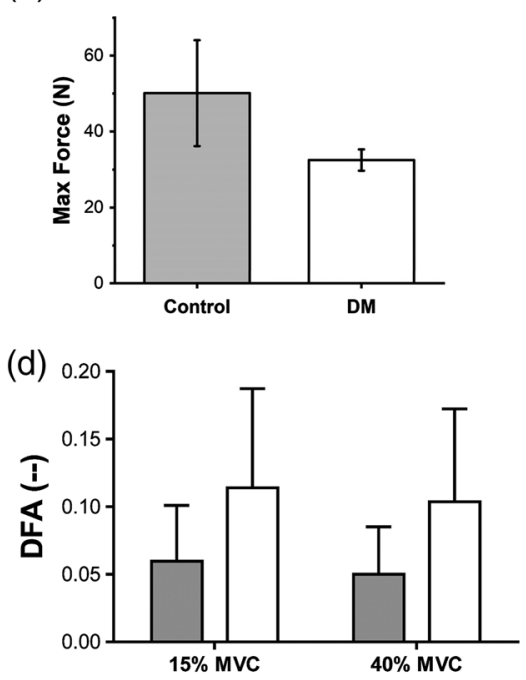

Fig. 3 Tactile sensory function data, maximal force data, and indices of submaximal force production. "Group" mean and SE values are shown. (a) Tactile sensory function data for each of the three nerves of the hand, (b) maximal pinch forces, (c) coefficient of variation (CV) in submaximal force production tasks, and (d) DFA in submaximal force production tasks. 
Gorniak et al.: Functional neuroimaging of sensorimotor cortices in postmenopausal women...

Table 2 Significant ANCOVA covariate output and regression results.

\begin{tabular}{|c|c|c|c|c|c|}
\hline Measure & Covariate & $F$ & $p$ Value & $r$ & $p$ Value \\
\hline \multirow[t]{4}{*}{ Tactile evaluation } & BMI & 11.68 & $<0.001$ & 0.29 & $<0.001$ \\
\hline & $A_{1 c}$ & 4.49 & $<0.05$ & 0.15 & $<0.05$ \\
\hline & PN status & 25.05 & $<0.001$ & 0.27 & $<0.001$ \\
\hline & Duration & 49.56 & $<0.001$ & - & - \\
\hline \multirow[t]{3}{*}{ RMSE } & $\% \mathrm{MVC}$ & 199.99 & $<0.001$ & - & - \\
\hline & BMI & 7.79 & $<0.01$ & - & - \\
\hline & PN status & 6.32 & $<0.05$ & - & - \\
\hline ApEn & HDL cholesterol & 4.02 & $<0.05$ & - & - \\
\hline \multirow[t]{2}{*}{$\mathrm{CV}$} & $\% \mathrm{MVC}$ & 2.033 & $<0.001$ & - & - \\
\hline & Total cholesterol & 4.29 & $<0.05$ & - & - \\
\hline \multirow[t]{3}{*}{ DFA } & BMI & 6.95 & $<0.05$ & - & - \\
\hline & Systole & 5.77 & $<0.05$ & - & - \\
\hline & Total cholesterol & 12.11 & $<0.001$ & -0.22 & 0.058 \\
\hline Digit $1 \mathrm{COP}$ & Menopausal age & 5.65 & $<0.05$ & - & - \\
\hline
\end{tabular}

Note: \%MVC refers to force production requirement level (15\% or $40 \% \mathrm{MVC}$ ); $\mathrm{A}_{1 \mathrm{c}}$ refers to glycated hemoglobin; ApEn refers to approximate entropy; BMI refers to body mass index; COP refers to center of pressure; $\mathrm{CV}$ refers to coefficient of variation; DFA refers to detrended fluctuation analysis; HDL cholesterol refers to high-density lipoprotein cholesterol; N/A refers to nonapplicable; PN status refers to diabetic peripheral neuropathy diagnosis; and systole refers to systolic blood pressure.

\subsection{Maximal Force Production}

The average force produced during pinch testing was $39.86 \pm 6.1 \mathrm{~N}$ across all participants [Fig. 3(b)]. Neither between group differences nor covariates emerged for maximal force production.

\subsection{Submaximal Force Production}

No group differences were found in RMSE or ApEn measures; instead, health state covariates provided significant effects, see Table 2 for details. Group effects found in CV $\left(F_{1,61}=4.50\right.$, $p<0.05)$ and DFA measures $\left(F_{1,61}=12.07, p<0.001\right)$, shown in Figs. 3(c) and 3(d), were replaced by health state covariates when they were added to the statistical model. Covariate results for CV and DFA are also included in Table 2. A finding of differences in contact area of digit 1 (thumb) utilized (via COP measurement ${ }^{2-24}$ ) was found with "menopausal age" $\left(F_{1,51}=5.65, p<0.05\right)$; however, no specific correlation between COP and menopausal age was explicitly found.

\subsection{Cortical Hemodynamic Responses}

\subsubsection{Cortical hemodynamic responses during sensory stimulation via vibration}

Average $\mathrm{HbO}$ and $\mathrm{HbR}$ data for the sensory stimulation task can be found in Figs. 4(a)-4(d). $\mathrm{HbO}$ did not show significant effects with respect to group $(p>0.5)$, hemisphere $(p>0.5)$, or region $(p>0.2)$. HbR did not show significant effects with respect to group $(p>0.8)$ or region $(p>0.2)$; however, a significant effect of hemisphere $\left(F_{1,11}=6.03, p<0.05\right)$ was found such that a larger increase in $\mathrm{HbR}$ was found for the left hemisphere as compared to the right 
Gorniak et al.: Functional neuroimaging of sensorimotor cortices in postmenopausal women...
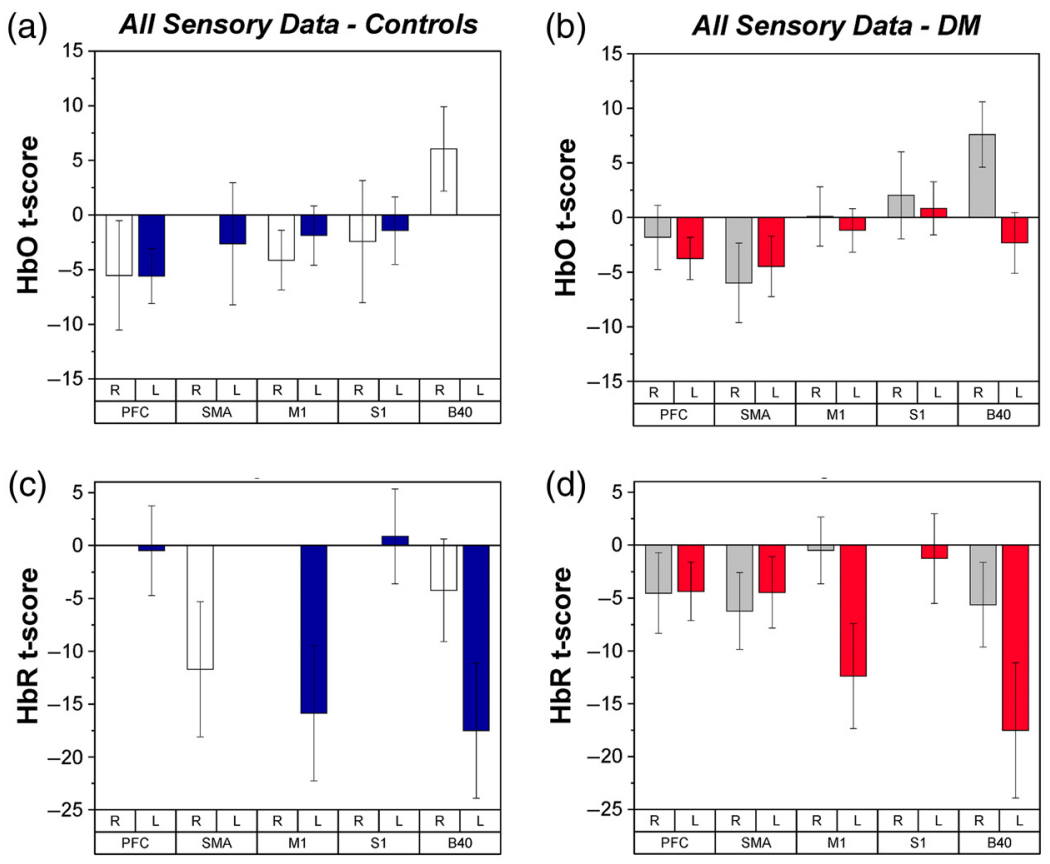

Fig. 4 fNIRS $t$-scores for $\mathrm{HbO}$ and $\mathrm{HbR}$ during vibrotactile stimulation for each group, as depicted by $\mathrm{ROI}$ and hemisphere. Mean and SE values are shown. (a) $\mathrm{HbO}$ of controls. White bars indicate right hemisphere and blue bars indicate left hemisphere. (b) $\mathrm{HbO}$ of PwDM. Gray bars indicate right hemisphere and red bars indicate left hemisphere. (c) HbR of controls. (d) HbR of PwDM.

Table 3 Significant correlation results for $\mathrm{HbO}$ and $\mathrm{HbR}$ measures.

\begin{tabular}{llrr}
\hline \hline Measure & \multicolumn{1}{c}{ Correlate } & $r$ & $p$ Value \\
\hline $\mathrm{HbO}$ (sensory stimulation) & Duration & -0.409 & $<0.005$ \\
& Prempro history & -0.325 & $<0.05$ \\
$\mathrm{HbR}$ (sensory stimulation) & PN status & -0.333 & $<0.05$ \\
& Prediabetes history & -0.313 & 0.055 \\
$\mathrm{HbO}$ (motor tasks) & $\mathrm{A}_{1 \mathrm{c}}$ & -0.44 & $<0.001$ \\
& ApEn & 0.185 & $<0.05$ \\
& BMl & -0.350 & $<0.001$ \\
$\mathrm{HbR}$ (motor tasks) & PN status & -0.252 & $<0.005$ \\
\hline \hline
\end{tabular}

Note: $A_{1 c}$ refers to glycated hemoglobin; ApEn refers to approximate entropy; $B M I$ refers to body mass index; $\mathrm{HbO}$ refers to oxygentated hemoglobin; $\mathrm{HbR}$ refers to deoxygentated hemoglobin; and PN status refers to diabetic peripheral neuropathy diagnosis.

hemisphere, consistent with stimulation of the right hand [Figs. 4(c) and 4(d)]. $\mathrm{HbO}$ and $\mathrm{HbR}$ values were found to correlate with some measures of health state, as reported in Table 3.

\subsubsection{Cortical hemodynamic responses during manual motor performance}

During the manual motor tasks, significant effects of group $\left(F_{1,106}=9.04, p<0.005\right)$ and a Hemisphere $\times$ Region interaction $\left(F_{4,106}=3.51, p<0.05\right)$ were found in $\mathrm{HbO}$, as shown in 

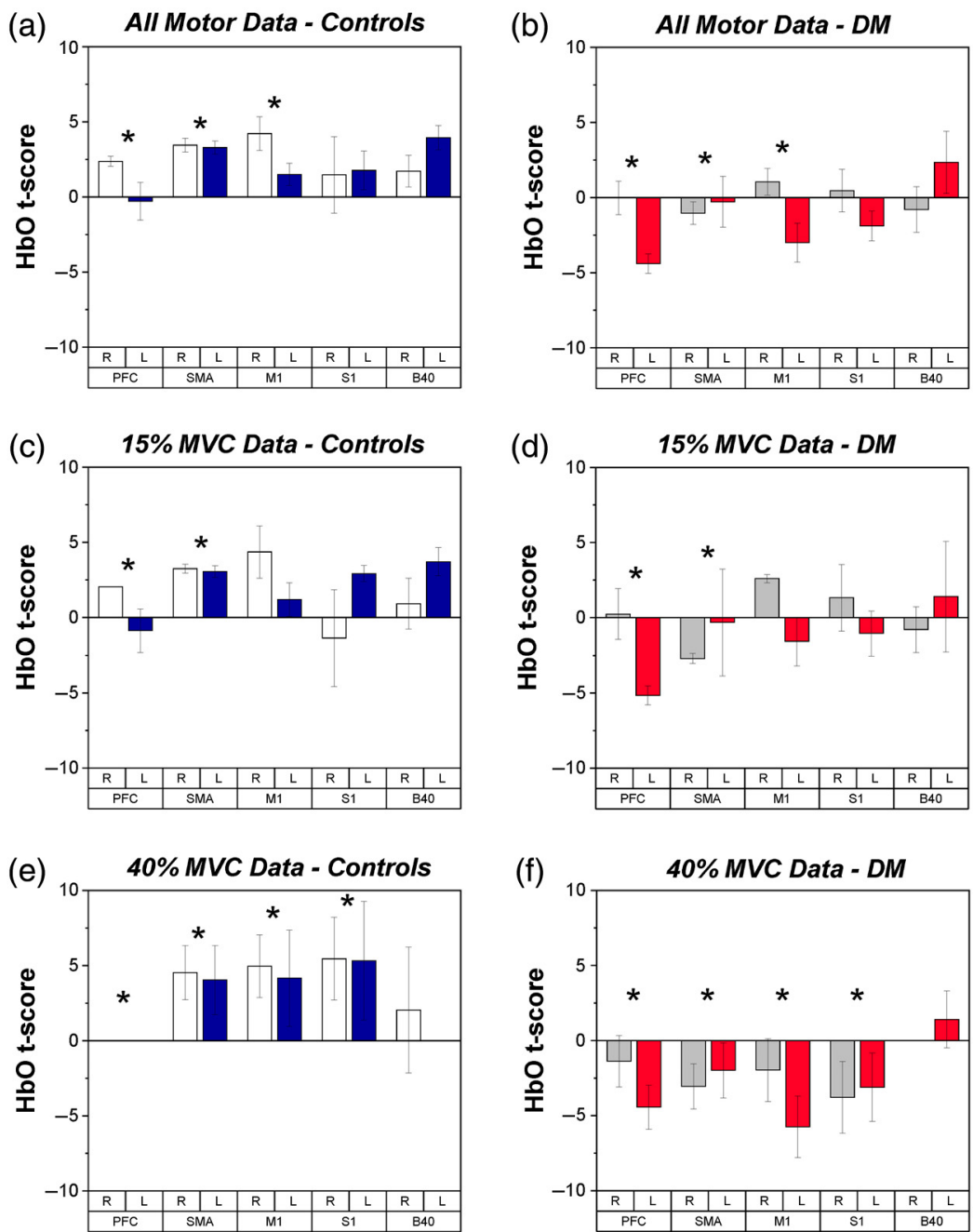

Fig. 5 fNIRS $t$-scores for HbO during submaximal force production tasks for each group, depicted by ROI and hemisphere. Mean and SE values are shown. * indicates a significant difference between groups. (a) $\mathrm{HbO}$ of controls across all motor tasks. White bars indicate right hemisphere and blue bars indicate left hemisphere. (b) $\mathrm{HbO}$ of PwDM across all motor tasks. Gray bars indicate right hemisphere and red bars indicate left hemisphere. (c) $\mathrm{HbO}$ of controls during $15 \% \mathrm{MVC}$ force production tasks. (d) $\mathrm{HbO}$ of PwDM during 15\% MVC force production tasks. (e) $\mathrm{HbO}$ of controls during $40 \%$ MVC force production tasks. (f) $\mathrm{HbO}$ of PwDM during $40 \% \mathrm{MVC}$ force production tasks.

Figs. 5(a) and 5(b). Overall, the data indicate significantly larger $\mathrm{HbO}$ values in controls versus PwDM, particularly in the left hemisphere during motor tasks. Notably, PFC, SMA, M1, and S1 were generally lower in terms of $\mathrm{HbO}$ in PwDM via Bonferroni corrected post-hoc. HbO values were found to correlate with some measures of health state and motor performance, as reported in Table 3.

Additional analyses of $\mathrm{HbO}$ indicated a significant difference in cortical $\mathrm{HbO}$ use between controls and PwDM, particularly in the low force production task (15\% MVC). Significant effects of group $\left(F_{1,39}=6.11, p<0.05\right)$ and a Hemisphere $\times$ Region interaction $\left(F_{4,39}=2.81\right.$, $p<0.05)$ were found in $\mathrm{HbO}$ during the $15 \%$ MVC task, as shown in Figs. 5(c) and 5(d). These effects are similar to that for all $\mathrm{HbO}$ data for the manual motor tasks. Notably, no major group, region, hemisphere effects, or interactions were found in the initial analysis of the $40 \%$ MVC task; however, an impact of menopausal age $\left(F_{1,32}=6.09, p<0.05\right)$ on $\mathrm{HbO}$ was found which helped reveal a group $\left(F_{1,32}=12.76, p<0.005\right)$ effect within the $40 \%$ MVC task, as shown in Figs. 5(e) and 5(f). While the correlation between menopausal age and HbO data was not significant, the models indicate a negative relationship between the two measures. 
Gorniak et al.: Functional neuroimaging of sensorimotor cortices in postmenopausal women...
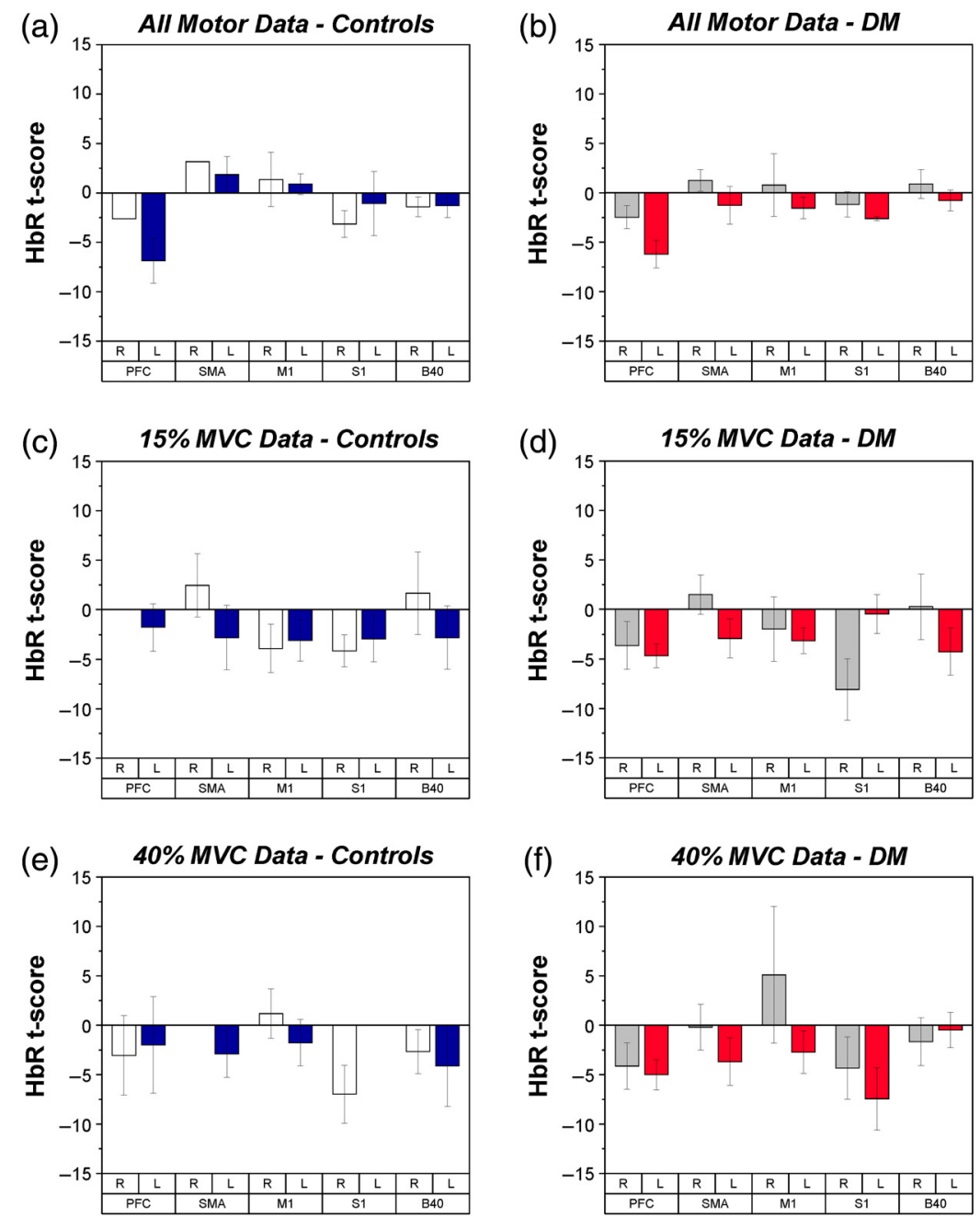

Fig. 6 fNIRS $t$-scores for HbR during submaximal force production tasks for each group, depicted by $\mathrm{ROI}$ and hemisphere. Mean and SE values are shown. (a) HbR of controls across all motor tasks. White bars indicate right hemisphere and blue bars indicate left hemisphere. (b) $\mathrm{HbR}$ of PwDM across all motor tasks. Gray bars indicate right hemisphere and red bars indicate left hemisphere. (c) HbR of controls during 15\% MVC force production tasks. (d) HbR of PwDM during 15\% MVC force production tasks. (e) HbR of controls during $40 \%$ MVC force production tasks. (f) $\mathrm{HbR}$ of PwDM during 40\% MVC force production tasks.

During the manual motor tasks, a significant effect of region $\left(F_{4,74}=2.66, p<0.05\right)$ was found in HbR, as shown in Fig. 6. Post-hoc analysis indicates that the PFC was overall more active than SMA, S1, and B40 during motor tasks. HbR values were found to correlate with some measures of health state, as reported in Table 3. Separate analyses of HbR by \%MVC did not yield any significant results.

\section{Discussion}

The purpose of the current study was to evaluate changes in cortical oxygenation indices of postmenopausal women both with and without DM during (1) tactile stimulation of the hands and (2) manual force production tasks. In terms of our hypotheses, the data support (at least in part) each of our two primary hypotheses. With respect to Hypothesis \#1, HbO values differed between groups during force production tasks while HbR did not show group effects in either vibrotactile stimulation or force production tasks. In support of Hypothesis \#2, decline in motor function output was observed in both tasks in PwDM as compared to controls; however, tactile 
detection thresholds did not differ between groups. With respect to our exploratory arm of the study, there is substantial evidence of the influence of poor health state as an impact on all measures of interest in this study. In the following paragraphs, we discuss the results of this study in regard to the published literature as it relates to cortical oxygenation and functional neuroimaging, possible sex-based differences in PwDM, and the impact of health state markers in assessment of both behavior and cortical function.

\subsection{Functional Cortical Activity and Tissue Oxygenation}

The primary outcome of this manuscript indicates a significant global difference in the use of $\mathrm{HbO}$ between postmenopausal female PwDM and age- and sex-matched controls. Specifically, cortical use of $\mathrm{HbO}$ was significantly higher in controls during the performance of motor tasks as compared to PwDM across most ROIs, suggesting that all aspects of sensorimotor function (including planning and executive function) may be directly impacted by DM. This finding coupled with simultaneous deficits in fine motor function in PwDM indicates a potential cortical root for motor performance degradation in PwDM. This is the first evidence of a cortical contribution for motor dysfunction in PwDM, whereas the traditional point of view is that peripheral nerve damage solely accounts for any motor deficits in this population. ${ }^{34-36}$ Interestingly, $\mathrm{HbO}$ differences in the fine motor function task (15\% MVC force production) suggest that tasks involving finer manual function control may be more negatively impacted by DM at the cortical level. This may have significant influence over the performance of activities of daily living (ADLs), as many ADLs are manual tasks involving low levels of force production and control.

Our finding of reduced cortical use of $\mathrm{HbO}$ is certainly notable, as $\mathrm{HbO}$ use is not indicated by other functional imaging techniques-most specifically functional magnetic resonance imaging (fMRI) - as they are not sensitive to $\mathrm{HbO}$. By its nature, $\mathrm{HbO}$ is diamagnetic (containing no unpaired electrons) and is thus not attracted to any magnetic field. Techniques, such as fMRI, rely on the paramagnetism of $\mathrm{HbR}$, thereby not fully representing potential issues with cortical hemodynamic responses, which involve both $\mathrm{HbO}$ and $\mathrm{HbR}$.

As cortical HbR has been shown to increase in proportion to manually produced forces, ${ }^{37,38}$ it is unlikely that the null $\mathrm{HbR}$ results in the motor tasks are due to the level of submaximal forces required in the current study. We acknowledge that $\mathrm{HbR}$ use has been found to increase during fatiguing tasks; ${ }^{39}$ currently, it is unknown if this phenomenon is consistent for PwDM. This provides an opportunity for immediate future investigation.

Reduced use of $\mathrm{HbO}$ in the cortex during motor tasks in PwDM may indicate reduced bioavailability of oxygen within the cortex. This observation is associated with the occurrence of motor impairment in the current data set. Currently, it is unclear as to why exactly this occurs. The evidence base indicates that DM is associated with increased hemoglobin-oxygen affinity, which is responsible for reduced oxygen delivery to tissues within the body ${ }^{40}$ However, DM is also associated with impaired hyperemic responses, endothelial dysfunction, and microvascular dysfunction. ${ }^{13,16,41,42}$ DM has also recently been linked to pathological neurovascular decoupling in PwDM (as reviewed in Ref. 43) and conflicting reports of reduced cerebral blood flow. ${ }^{44,45}$ There is the possibility that impaired active hyperemia and changes in overall blood oxygenation both contribute to the deficits we have observed in PwDM. However, this cannot be resolved by the current data set, but the data do suggest that further mechanistic investigation is warranted into the neurovascular and potential reduced blood flow roots of reduced cortical $\mathrm{HbO}$ in female PwDM.

\subsection{Influence of Sex on Cortical Structure/Function}

Contrary to our previous findings, ${ }^{5,12}$ differences in tactile function and maximal force profiles between the current sample of postmenopausal PwDM and age- and sex-matched controls were not found. On the surface, this result is surprising; however, this particular study focused on postmenopausal women, whereas our previous work included a cross-section of participant ages, including both males and females. It is possible that sex-based differences in sensorimotor function exist in PwDM, particularly as age increases, as supported by Ref. 33. Sex-based difference 
in the presentation of DM complications is an emerging area of interest in metabolic research. ${ }^{46}$ Our recent work indicates a significant impact of sex, age, handedness, and metabolic health on the development of poor tactile sensation. ${ }^{33}$ While the presence of DM and PN does adversely impact overall tactile function, sex-based differences continue to persist ${ }^{33}$ such that females present with less impairment in behavioral measures as compared to males, reinforcing the behavioral results within the current data set. The root of these sex-based differences may stem from several sources, including differences in sex hormone levels (e.g., testosterone, estrogen, and other androgens), sexual dimorphism and asymmetry of the cortex, ${ }^{47-49}$ and reports of persistent metabolic youth in the aging female brain as compared to males. ${ }^{50}$ Reduced cortical asymmetry in females may also partially explain the absence of strong hemispheric differences in hemodynamic responses within the current data set. ${ }^{47}$

Decreases in hyperemic responses have also been found in postmenopausal females as compared to males (both without DM), indicating a baseline difference in blood flow due to sex, which may impact both sensory and motor functions. ${ }^{51-53}$ There is some evidence that estrogenbased hormone replacement therapy (HRT) improves hyperemic responses in postmenopausal females $;^{54,55}$ but there is no indication that it is protective against deficits induced by DM. Discontinued use of conjugated estrogen HRT in the early 2000s due to cardiovascular complications of the treatment (e.g., Prempro) further complicates the picture. ${ }^{56}$ Specifically, Prempro use introduces significant additional cardiovascular disease risk in females. In the current data set, reduced cortical oxygenation along with motor deficits was demonstrated in PwDM as compared to controls. These results were not impacted by HRT status (both HRT history overall and specifically by Prempro use), indicating cortical deficits linked to DM without a directional influence of HRT in postmenopausal females.

\subsection{Influence of Health State on Behavioral and Cortical Activation Measures}

Additionally, significant influences of overall poor health state were found across the data set. Specifically, measures such as high $\mathrm{A}_{1 \mathrm{c}}$, high blood pressure, high BMI, and high cholesterol, appeared as detrimental factors in the current data set. This suggests that overall cardiovascular health may be a significant factor in the interplay between cortical activity and overt sensorimotor behaviors. We acknowledge that a history of PN did appear as an influence in some of the statistical models; however, it did not account for the between-group differences found; instead, it enhanced the effects. This suggests that nonperipheral factors do indeed relate to sensorimotor dysfunction in PwDM contrary to prior clinical points of view. ${ }^{34-36}$ Overall, our data indicate a negative impact of poor health state, independent of specific DM medical management, that is a significant contributor to reduced functional use of $\mathrm{HbO}$ by the cortex.

\subsection{Limitations}

We acknowledge that the present study does not account for systemic changes that may have occurred within the data set [short-term physiological changes (e.g., respiration)]. Despite our instructions to breathe normally, it is possible that during the motor tasks, participants held their breath while exerting the force, which may have impacted the fNIRS signals. In future work, we recommend adding additional measures of blood oxygenation and respiration rates in order to evaluate this possibility.

\subsection{Conclusion}

Overall, the data demonstrate reduced use of cortical HbO in PwDM while they simultaneously showed deficits in manual motor tasks, providing the first evidence of functional cortical activity deficits relating to motor dysfunction in this population. Functional cortical activation deficits were not specifically noted in HbR. Similar effects were not found in evaluation of sensory function. Health state indices were found to clarify group effects. Further work is needed to clarify potential sex-based differences in PwDM and the root of reduced cortical oxygenation use in PwDM during motor tasks. 


\section{Disclosures}

None of the authors has any conflict of interest to disclose.

\section{Acknowledgments}

This work was supported by American Heart Association (AHA) Grant No. 16BGIA27250047 to SLG. AHA had no role in the design of the study, collection/analysis/interpretation of data, nor the writing/submission of this article.

\section{Code, Data, and Materials Availability}

Data analyzed in this project will be available via Zenodo (https://zenodo.org/) upon manuscript publication. As public sharing of protected health information, such as date of birth, date of diagnosis, dates of treatment, and other private medical history information pertinent to each participant, may violate HIPAA and the Texas Medical Privacy Act, these data will not be shared to protect patient identity.

\section{References}

1. Centers for Disease Control and Prevention, "National Diabetes Statistics Report," http:// www.cdc.gov/diabetes/pubs/factsheet11.htm?loc=diabetes-statistics (2020).

2. E. van den Berg et al., "Cognitive functioning in elderly persons with type 2 diabetes and metabolic syndrome: the Hoorn Study," Dementia Geriatr Cognit Disord 26(3), 261-269 (2008).

3. A. L. Christman et al., "Cranial volume, mild cognitive deficits, and functional limitations associated with diabetes in a community sample," Arch. Clin. Neuropsychol. 25(1), 49-59 (2010).

4. S. L. Gorniak et al., "Cognitive-motor impairment in manual tasks in adults with type 2 diabetes," OTJR 40(2), 113-121 (2019).

5. S. L. Gorniak et al., "Detecting subtle fingertip sensory and motor dysfunction in adults with type II diabetes,” Exp. Brain Res. 232(4), 1283-1291 (2014).

6. G. J. Snoek et al., "Survey of the needs of patients with spinal cord injury: impact and priority for improvement in hand function in tetraplegics," Spinal Cord 42(9), 526-532 (2004).

7. K. D. Anderson, "Targeting recovery: priorities of the spinal cord-injured population," J. Neurotrauma 21(10), 1371-1383 (2004).

8. J. E. Casanova, J. S. Casanova, and M. J. Young, "Hand function in patients with diabetes mellitus," South. Med. J. 84(9), 1111-1113 (1991).

9. R. I. Cederlund et al., "Hand disorders, hand function, and activities of daily living in elderly men with type 2 diabetes," J. Diabetes Complications 23(1), 32-39 (2009).

10. A. A. Sayer et al., "Type 2 diabetes, muscle strength, and impaired physical function: the tip of the iceberg?" Diabetes Care 28(10), 2541-2542 (2005).

11. M. D. Allen et al., "Length dependent loss of motor axons and altered motor unit properties in human diabetic polyneuropathy," Clin. Neurophysiol. 125(4), 836-843 (2014).

12. N. Ochoa, G. R. Gogola, and S. L. Gorniak, "Contribution of tactile dysfunction to manual motor dysfunction in type II diabetes," Muscle Nerve 54, 895-902 (2016).

13. L. Pollonini et al., "Hemodynamic function of forearm muscle in older adult females with type II diabetes," J. Aging Phys. Activity 21, 1-8 (2020).

14. D. W. Zochodne, "Diabetes mellitus and the peripheral nervous system: manifestations and mechanisms," Muscle Nerve 36(2), 144-166 (2007).

15. J. Petrofsky, S. Lee, and S. Bweir, "Gait characteristics in people with type 2 diabetes mellitus," Eur. J. Appl. Physiol. 93(5-6), 640-647 (2005).

16. J. S. Petrofsky, "The effect of type-2-diabetes-related vascular endothelial dysfunction on skin physiology and activities of daily living," J. Diabetes Sci. Technol. 5(3), 657-667 (2011). 
Gorniak et al.: Functional neuroimaging of sensorimotor cortices in postmenopausal women...

17. A. Kautzky-Willer, J. Harreiter, and G. Pacini, "Sex and gender differences in risk, pathophysiology and complications of type 2 diabetes mellitus," Endocr. Rev. 37(3), 278-316 (2016).

18. V. Raparelli et al., "Sex differences in type-2 diabetes: implications for cardiovascular risk management," Curr. Pharm. Des. 23(10), 1471-1476 (2017).

19. R. C. Oldfield, "The assessment and analysis of handedness: the Edinburgh inventory," Neuropsychologia 9(1), 97-113 (1971).

20. Y. Feng, F. J. Schlösser, and B. E. Sumpio, "The Semmes Weinstein monofilament examination as a screening tool for diabetic peripheral neuropathy," J. Vasc. Surg. 50(3), 675-682.e1 (2009).

21. N. Ochoa and S. L. Gorniak, "Changes in sensory function and force production in adults with type II diabetes," Muscle Nerve 50(6), 984-990 (2014).

22. V. Zatsiorsky, Kinetics of Human Motion, 1st ed., Human Kinetics, Champaign, Illinois (2002).

23. M. Duarte and V. M. Zatsiorsky, "Effects of body lean and visual information on the equilibrium maintenance during stance," Exp. Brain Res. 146(1), 60-69 (2002).

24. S. L. Gorniak and J. L. Alberts, "Effects of aging on force coordination in bimanual task performance," Exp. Brain Res. 229(2), 273-284 (2013).

25. R. Oostenveld and P. Praamstra, "The five percent electrode system for high-resolution EEG and ERP measurements," Clin. Neurophysiol. 112(4), 713-719 (2001).

26. C. M. Aasted et al., "Anatomical guidance for functional near-infrared spectroscopy: AtlasViewer tutorial," Neurophotonics 2(2), 020801 (2015).

27. G. E. Strangman, Z. Li, and Q. Zhang, "Depth sensitivity and source-detector separations for near infrared spectroscopy based on the Colin27 brain template," PLoS One 8(8), e66319 (2013).

28. M. Cope and D. T. Delpy, "System for long-term measurement of cerebral blood and tissue oxygenation on newborn infants by near infra-red transillumination," Med. Biol. Eng. Comput. 26(3), 289-294 (1988).

29. D. T. Delpy et al., "Estimation of optical pathlength through tissue from direct time of flight measurement," Phys. Med. Biol. 33(12), 1433-1442 (1988).

30. J. W. Barker, A. Aarabi, and T. J. Huppert, "Autoregressive model based algorithm for correcting motion and serially correlated errors in fNIRS," Biomed. Opt. Express 4(8), 1366-1379 (2013).

31. H. Santosa et al., "The NIRS brain AnalyzIR toolbox," Algorithms 11(5), 73 (2018).

32. T. J. Huppert, "Commentary on the statistical properties of noise and its implication on general linear models in functional near-infrared spectroscopy," Neurophotonics 3(1), 010401 (2016).

33. S. L. Gorniak et al., "Sex-based differences and aging in tactile function loss in persons with type 2 diabetes," PLoS One (under review) (2020).

34. S. M. Manschot et al., "Peripheral and central neurologic complications in type 2 diabetes mellitus: no association in individual patients," J. Neurol. Sci. 264(1-2), 157-162 (2008).

35. A. I. Vinik, C. Casellini, and M. Nevoret, "Diabetic neuropathies," in Endotext, pp. 1-93, MD Text, Inc., South Dartmouth, Massachusetts (2011).

36. A. I. Vinik et al., "Diabetic neuropathy in older adults," Clin. Geriatr. Med. 24(3), 407-435 (2008).

37. B. Keisker et al., "Differential force scaling of fine-graded power grip force in the sensorimotor network," Hum. Brain Mapp. 30(8), 2453-2465 (2009).

38. A. Sterr et al., "fMRI effects of task demand and feedback accuracy on grip force tracking," Neurosci. Lett. 457(2), 61-65 (2009).

39. Z. Jiang et al., "Strengthened functional connectivity in the brain during muscle fatigue," Neuroimage 60(1), 728-737 (2012).

40. L. J. Pu et al., "Increased blood glycohemoglobin $A_{1 c}$ levels lead to overestimation of arterial oxygen saturation by pulse oximetry in patients with type 2 diabetes," Cardiovasc. Diabetol. 11, 110 (2012).

41. A. L. Barwick et al., "Peripheral sensory neuropathy is associated with altered postocclusive reactive hyperemia in the diabetic foot," BMJ Open Diabetes Res. Care 4(1), e000235 (2016). 
Gorniak et al.: Functional neuroimaging of sensorimotor cortices in postmenopausal women...

42. M. F. Meyer et al., "Impaired flow-mediated vasodilation in type 2 diabetes: lack of relation to microvascular dysfunction," Microvasc. Res. 76(1), 61-65 (2008).

43. M. Coucha et al., "Impact of metabolic diseases on cerebral circulation: structural and functional consequences," Compr. Physiol. 8(2), 773-799 (2018).

44. L. Hunt et al., "Cerebral blood flow abnormalities in brain regions responsible for cognitive function in type 2 diabetes," Diabetes 67(Suppl. 1), 1798-P (2018).

45. J. F. A. Jansen et al., "Cerebral blood flow, blood supply, and cognition in type 2 diabetes mellitus," Sci. Rep. 6(1), 10 (2016).

46. A. Norhammar and K. Schenck-Gustafsson, "Type 2 diabetes and cardiovascular disease in women," Diabetologia 56(1), 1-9 (2013).

47. A. Wisniewski, "Sexually-dimorphic patterns of cortical asymmetry, and the role for sex steroid hormones in determining cortical patterns of lateralization," Psychoneuroendocrinology 23(5), 519-547 (1998).

48. D. E. Adamo, S. Scotland, and B. J. Martin, "Upper limb kinesthetic asymmetries: gender and handedness effects," Neurosci. Lett. 516(2), 188-192 (2012).

49. K. Amunts et al., "Interhemispheric asymmetry of the human motor cortex related to handedness and gender," Neuropsychologia 38(3), 304-312 (2000).

50. M. S. Goyal et al., "Persistent metabolic youth in the aging female brain," Proc. Natl Acad. Sci. U.S.A. 116(8), 3251-3255 (2019).

51. B. A. Parker et al., "Sex-specific influence of aging on exercising leg blood flow," J. Appl. Physiol. 104, 655-664 (2008).

52. B. A. Parker et al., "Evidence for reduced sympatholysis in leg resistance vasculature of healthy older women," Am. J. Physiol. 292(2), H1148-H1156 (2007).

53. D. N. Proctor et al., "Impaired leg vasodilation during dynamic exercise in healthy older women," J. Appl. Physiol. 95(5), 1963-1970 (2003).

54. L. R. Peterson et al., "Estrogen increases hyperemic microvascular blood flow velocity in postmenopausal women," J. Gerontol. A 55(3), M174-M179 (2000).

55. P. J. Fadel et al., "Augmented sympathetic vasoconstriction in exercising forearms of postmenopausal women is reversed by oestrogen therapy," J. Physiol. 561(3), 893-901 (2004).

56. G. Wells and D. M. Herrington, "The Heart and Estrogen/Progestin Replacement Study: what have we learned and what questions remain?" Drugs Aging 15(6), 419-422 (1999).

Stacey L. Gorniak is currently an associate professor in the Department of Health and Human Performance at the University of Houston. She received her PhD from Penn State in 2009 and completed her postdoctoral fellowship at Cleveland Clinic in 2011. She is the current director of the Center for Neuromotor and Biomechanics Research. Her research interests include understanding the central and peripheral effects of type II diabetes on cognitive-sensorimotor function.

Victoria E. Wagner received her PhD from the University of Houston. She is an assistant professor at The University of St.Thomas in Houston, Texas. Her research interests focus on speech perception and production as well as brain changes associated with expertise.

Kelly Vaughn is currently an assistant professor of pediatrics at the Children's Learning Institute at the University of Texas Health Science Center at Houston. She earned her doctorate degree in Developmental Cognitive Neuroscience at the University of Houston. Her research broadly focuses on supporting the development of bilingual children (i.e., children who are acquiring two languages). She uses neuroimaging and cognitive assessments to understand typical and atypical bilingual development.

Jonathan Perry graduated from the University of Houston with a BS degree in kinesiology. While working on his undergraduate degree, he was also a part of the Laboratory of Integrated Physiology, where he worked under Dr. Yunjung Park on mouse models in Alzheimer's disease. He then moved into the College of Technology to work on his MS degree in computational health informatics under Dr. Luca Pollonini with focused research on fNIRS. 
Lauren Gulley Cox received her PhD in kinesiology from the University of Houston, Houston, Texas, in 2020. Her research interests focus on understanding neuromuscular control and biomechanics and how these are affected due to aging, disease, and injury.

Arturo E. Hernandez is currently a professor in the Department of Psychology at the University of Houston. He is the director of the Laboratory for the Neural Bases of Bilingualism. He received his $\mathrm{PhD}$ from the University of California at San Diego. His research interests are focused on the nature of language processing using both behavioral and neuroimaging methods.

Luca Pollonini is an assistant professor at the University of Houston, Texas. His research interests are in the development of instruments and methods for measuring tissue hemodynamics using optical near-infrared spectroscopy and diffusion optical imaging as well as in their clinical translation. He is a member of the Society for Functional Near Infrared Spectroscopy and an associate editor of the IEEE Journal of Translational Engineering in Health and Medicine. 